\title{
Information Requirements under the Essential-Use Concept: PFAS Case Studies
}

\author{
Juliane Glüge, Rachel London, Ian T. Cousins, Jamie DeWitt, Gretta Goldenman, Dorte Herzke, \\ Rainer Lohmann, Mark Miller, Carla A. Ng, Sharyle Patton, Xenia Trier, Zhanyun Wang, \\ and Martin Scheringer*
}

Cite This: https://doi.org/10.1021/acs.est.1c03732

Read Online

ACCESS | Lلll Metrics \& More | 回 Article Recommendations | SI Supporting Information

ABSTRACT: Per- and polyfluoroalkyl substances (PFAS) are a class of substances for which there are widespread concerns about their extreme persistence in combination with toxic effects. It has been argued that PFAS should only be employed in those uses that are necessary for health or safety or are critical for the functioning of society and where no alternatives are available ("essential-use concept"). Implementing the essential-use concept requires a sufficient understanding of the current uses of PFAS and of the availability, suitability, and hazardous properties of alternatives. To illustrate the information requirements under the essential-use concept, we investigate seven different PFAS uses, three in

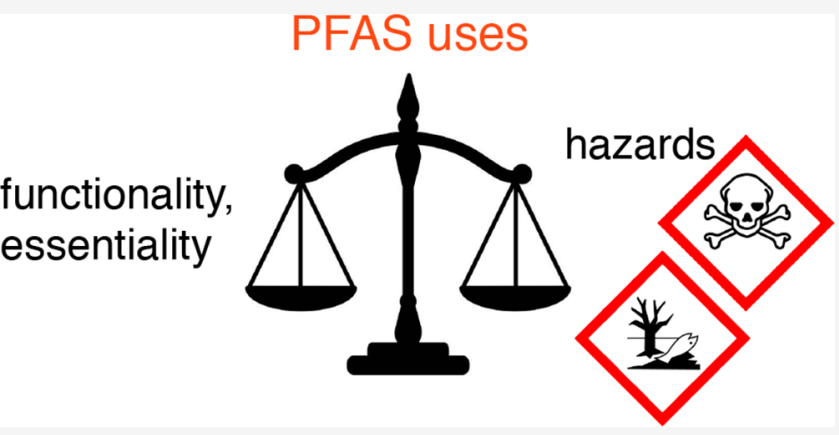
consumer products and four industrial applications. We investigate how much information is available on the types and functions of PFAS in these uses, how much information is available on alternatives, their performance and hazardous properties and, finally, whether this information is sufficient as a basis for deciding on the essentiality of a PFAS use. The results show (i) the uses of PFAS are highly diverse and information on alternatives is often limited or lacking; (ii) PFAS in consumer products often are relatively easy to replace; (iii) PFAS uses in industrial processes can be highly complex and a thorough evaluation of the technical function of each PFAS and of the suitability of alternatives is needed; (iv) more coordination among PFAS manufacturers, manufacturers of alternatives to PFAS, users of these materials, government authorities, and other stakeholders is needed to make the process of phasing out PFAS more transparent and coherent.

KEYWORDS: PFAS, essential use, chrome plating, fluoropolymer, carpet

\section{INTRODUCTION}

Per- and polyfluoroalkyl substances (PFAS) are a class of thousands of substances ${ }^{1,2}$ most of which are either persistent themselves or are transformed into persistent compounds in the environment. Further, the few PFAS studied to date have shown a wide range of biological activity in cell-based, animal, and human epidemiological studies. ${ }^{3}$ Because of these concerns, it has been argued that PFAS should only be employed in those uses that are necessary for health, safety, or are critical for the functioning of society and where no alternatives are available. ${ }^{4-6}$ This concept of "essential uses" has been incorporated into a working paper on "Elements for an EU strategy for PFAS"7 and also into the European Commission's Chemicals Strategy for Sustainability as well as the accompanying "Commission Staff Working Document Poly- and Perfluoroalkyl Substances (PFAS)".

Implementing the essential-use concept requires a sufficient understanding of the current uses of PFAS and of the availability, suitability, and hazardous properties of alternatives. Cousins et al. (2019a) ${ }^{4}$ analyzed nine different use areas of PFAS and the availability of alternatives. Other research groups and institutions have also looked into alternatives to PFAS in certain use areas, $^{9-15}$ and additional information is available on the OECD Portal on PFAS. ${ }^{16}$ Glüge et al. $(2020)^{17}$ characterized more than 200 uses of more than 1400 individual PFAS. This work has shown that PFAS uses are very diverse, and this diversity is a challenge to the implementation of the essential-use concept. In many cases, it is not clear whether the use of PFAS is essential. Accordingly, there is a need for a better understanding of where PFAS are used, what their specific functions in these uses are, and how easy or difficult it is to find alternatives.

To address this need, we investigate here a set of seven different PFAS uses, three in consumer products and four

Special Issue: Emerging Contaminants: Fluorinated

Alternatives to Existing PFAS Compounds

Received: June 7, 2021

Revised: August 25, 2021

Accepted: September 12, 2021 
industrial applications. We investigate how much information is available on the types and functions of PFAS in these uses and, if needed, how much information is available on alternatives, their performance and hazardous properties, and finally, whether this information is sufficient as a basis for deciding on the essentiality of a PFAS use. On this basis, we aim to make a contribution to a broader understanding of the information requirements of the essential-use concept and to illustrate the type of questions that are brought up for decision makers by the essential-use concept.

\section{METHODS}

2.1. Selection of Case Studies. The intention of the case studies selected is to cover a range of different PFAS uses from relatively simple to more complex cases for which there is at least some information on alternatives available in the public domain. By looking into PFAS uses in consumer products and in industrial processes, the diversity of PFAS uses is reflected, and different types of assessment tasks and decision-making situations are illustrated. In addition, several of the case studies are in areas where PFAS have caused serious contamination problems, for example, fluoropolymer ${ }^{17-20}$ or semiconductor production. $^{21,22}$

Given the large number of PFAS and PFAS uses, ${ }^{16}$ the seven case studies are a limited selection. Nevertheless, they show an interesting and relevant range of assessment tasks that may be typical for future applications of the essential-use concept. In particular, the industrial uses may deserve more discussion and further analysis.

The three case studies of PFAS uses in consumer products are on PFAS in bicycle lubricants, carpets, and cleaning products. The four industrial uses of PFAS are in fluoropolymer production, in the semiconductor industry, in chrome plating, and in chemical-driven oil production. For each case study, an extensive search of the available literature was conducted, including peer-reviewed journal articles, monographs, industry reports, product descriptions, and patents. In addition, we contacted PFAS manufacturers and downstream users and received additional input from technical experts for most of the case studies, in particular for bicycle lubricants, chrome plating, fluoropolymer production, and PFAS uses in the semiconductor industry. Details of the contacted businesses are provided in the Supporting Information (SI-1, Table S4).

2.2. Alternatives Assessment. Alternatives for each of the PFAS uses in the seven case studies were mainly found through literature searches. Confirmation of the information was obtained through discussions with providers of alternatives. Alternatives for which chemical identification information (CAS number or SMILES code) was found are listed in the accompanying MS Excel document "Gluege_et_al_SI-2.xlsx" (SI-2). In some cases, the chemical identity of alternatives was declared as confidential business information and not shared.

Where the chemical identity of the alternatives could be determined, their human-health and environmental hazards were compared with those of the PFAS currently used. Data on the hazards were taken from three sources: the ECHA Classification \& Labeling (C\&L) Inventory, the ECHA $\mathrm{REACH}$ registration database, and the EPI Suite estimation tool, ${ }^{23}$ where appropriate. Where a charged organic substance was identified, a neutral form of the substance was run through EPI Suite. This may introduce error into the calculation. Data were collected or generated for the following hazards: carcinogenicity, mutagenicity, reproductive toxicity (human health) and persistence, bioaccumulation, ecotoxicity, and mobility (environment). The data collected are provided in the SI-2.

When a brand name is mentioned in this work, this does not constitute endorsement of the brand or its product. The chemical identity of the PFAS in the different uses was taken from Glüge et al. (2020). ${ }^{16}$

In addition to cases where an alternative is a direct replacement of PFAS by other chemicals with the same technical function, there are also cases where the design of the product or technical process can be modified such that the technical need for PFAS no longer exists. Depending on the chemicals used in the modified products or processes (if any), an alternative assessment may or may not be needed.

2.3. Essentiality Categories. Where possible, we applied the categories of "non-essential", "substitutable", and "essential" uses, as defined by Cousins et al. (2019a), ${ }^{4}$ to the PFAS identified in the seven case studies. We did not judge whether or not a product or process is essential for health, safety or the functioning of society. Instead, the classification is based on whether the function provided by PFAS is needed in a specific product or process and on the availability of suitable alternatives. Products or processes where PFAS are not needed at all are classified as "non-essential". Products or processes where PFAS can be replaced by other substances or processes are classified as "substitutable". Cases where PFAS cannot (yet) be replaced are classified as essential. A further discussion and development of criteria for essential uses is not part of this work.

\section{CASE STUDIES ON ALTERNATIVES TO PFAS}

3.1. Bicycle Lubricants. Uses. Polytetrafluoroethylene (PTFE) is added to different kinds of bicycle lubricants including dry lubes, wet lubes, and wax lubes. Dry lubes are designed for riding in dry conditions and are often made by mixing ca. $10 \%$ lubricant (synthetic oils and additives) and $90 \%$ carrier fluid (solvent). Wet lubes contain larger quantities of higher-viscosity synthetic oils and additives such as PTFE and are intended for riding in wet conditions. ${ }^{24}$ There are also lubricants based on paraffin wax mixed with additives such as PTFE and a carrier fluid. PTFE may be used within a range of $5-25 \%$ by weight in dry lubes to reduce friction and wear. ${ }^{25}$ Wet lubricants seem to contain less PTFE; the safety data sheet of a wet PTFE lubricant reveals a PTFE content of $1-3 \%$ by weight. ${ }^{26}$ Another aspect is that a PTFE content of less than $1 \%$ may be added just for marketing purposes. ${ }^{27}$ For a substantial effect on performance, at least $4-5 \%$ PTFE is needed. $^{27}$

Availability of Alternatives and Alternatives Assessment. There are lubricants on the market that do not contain PTFE and perform well according to tests and user experiences. ${ }^{28-30}$ The providers of alternative lubricants state that their lubricants are "plant based" and decompose rapidly, ${ }^{31}$ but no information about biodegradability test results is provided. However, it can be assumed that, compared to the extreme persistence of PTFE, the persistence of these alternatives is much lower.

Conclusion. Although PFAS in bicycle lubricants may increase lubricating performance, they are technically not needed to keep chains lubricated. In addition, PFAS-free products have always been available on the market. In 
conclusion, the use of PFAS in bicycle lubricants is nonessential.

3.2. Carpets. Uses. PFAS have been used in carpets to impart water and oil repellency, stain resistance, and soil release to synthetic carpet face fibers. ${ }^{32}$ The reason is that nylon and other synthetic fibers are both oleophilic and hydrophobic and, thus, have a great affinity for soils. Soil removal on untreated nylon is therefore more difficult than on treated nylon.

Availability of Alternatives. One carpet manufacturer, Interface, changed the formulation of the nylon used in face fibers so that the fibers themselves are stain-resistant. ${ }^{33}$ The yarn producer, Aquafil, offers stain-free fibers based on the nylon-6 fiber "Econyl StayClean". ${ }^{44}$ This fiber is made from nylon waste that was depolymerized and recycled. ${ }^{35}$ No PFAS are added to the recycled fiber. Another yarn manufacturer, Universal Fibers, describes the use of a "sulfonated nylon copolymer" for a PFAS-free stain protection. ${ }^{32}$ In this case PFAS are not needed because the modified nylon fiber is stainresistant itself. The carpet manufacturer, Tarkett, on the other hand, treats its "Tandus Centiva"-branded products with fluorine-free soil protection products, ${ }^{36}$ which means that in the topical treatment PFAS are replaced by other chemicals.

Some PFAS-free carpets can also be found under the "Blue Angel" certificate. The certificates Blue Angel, ${ }^{37}$ Nordic Swan and Cradle-to-Cradle ${ }^{38}$ ban halogenated organic compounds (including all PFAS) from their labeled products. GUT (Association for Environmentally Friendly Carpets) lists 33 single PFAS that are not allowed in products with their certificate $^{39}$ and Oeko-Tex 100 bans PFOA-related substances. $^{40}$ In response to our information request, Blue Angel replied that PFAS-free polyamide carpets have been registered by the Condor Group. ${ }^{41}$

Alternatives Assessment. Limited information about alternatives is publicly available. ${ }^{32}$ Some information on chemical synthesis processes and ingredients can be found in patents, as listed in the SI- 1 . However, no information is available on the specific properties of the materials or on any results from toxicity or degradability tests. According to the manufacturers contacted, this is confidential business information.

Conclusion. It is possible and economically feasible to produce synthetic carpets without PFAS-based impregnation. PFAS in carpets are therefore substitutable. More information on the alternatives should be made publicly available so that their impacts can be evaluated transparently.

3.3. Cleaning Products. Uses. PFAS lower the surface tension and improve wetting and rinse-off in a variety of industrial and household cleaning products. Examples are car wash products, floor cleaning products and floor polish, carpet spot cleaner, cleaning solutions for optical devices, and dishwashing liquids in which, for example, PFOA has been detected. $^{42,43}$

Availability of Alternatives. Alternative household cleaning products are based on ingredients that are biodegradable, in some cases readily biodegradable. These products are available on the market. Examples for surfactants other than PFAS used in dish soap include for example sodium lauryl sulfate and lauryl glucoside. ${ }^{44}$ Surfactants used in laundry detergent are for example $\mathrm{C} 12-\mathrm{C} 16$ pareth-7, potassium cocoate, decyl glucoside $^{45}$ or sophorolipids. ${ }^{46}$ Examples for floor polish and carpet spot cleaner are provided in the SI-1.
Alternatives Assessment. The list of ingredients of a dish soap (Ecover Zero dish soap) ${ }^{44}$ was used for the assessment of alternatives. No indication of carcinogenic, mutagenic, or reproductive hazards was found for any of the ingredients. The environmental hazards were also lower: all substances in the dishwashing liquid have calculated degradation half-lives of 437 days, with biodegradation studies available and in agreement with the estimated values for most of the substances. Regarding bioaccumulation, all logarithmic octanol-water partition coefficients $\left(\log K_{\mathrm{OW}}\right)$ are below 3 , and all bioconcentration factors (BCFs) are below 20. Not all ingredients are listed in the REACH database, but data on the BCF for those available agreed with the estimated values. The use of these substances represents therefore a reduced human-health and environmental hazard when compared to the PFAS used in cleaning products. However, for some surfactants used in floor polish and carpet spot cleaner, the data in the $C \& L$ inventory indicate that they are toxic to aquatic organisms.

Conclusion. PFAS in household cleaning products are not technically needed; many alternatives are available and PFAS uses are non-essential. For industrial cleaning products, it would be necessary to look at each case individually in order to evaluate what level of performance is needed, and why, and if alternatives to PFAS are available.

3.4. Chrome Plating. Uses. PFAS are used in chrome plating as wetting agents in the pretreatment (etching) of plastic and as mist suppressing agents in the electroplating process. In electroplating (often referred to as chrome plating), fluorosurfactants reduce the exposure to toxic hexavalent chromium aerosols from the plating baths by (i) reducing the size of the bubbles formed and (ii) forming a barrier over the electrolyte solution. More information about PFAS uses in both processes is provided in the SI-1.

Fluorinated surfactants have been used previously for both decorative chrome plating and hard chrome plating. Hard chrome plating provides resistance against corrosion and abrasion to various items such as hydraulic cylinders and rods or railroad wheel bearings and couplers. ${ }^{47}$ Decorative chrome plating is mainly used for plated parts, for example in the automotive industry (including car and truck bumpers), but also in the sanitary, medicine, cosmetic, and furniture sectors. $^{47,48}$

Availability of Alternatives. Tests showed that chromium(VI) emissions can be reduced substantially by avoiding air convection. $^{49}$ Some companies use closed systems with underpressure and found almost no chromic-acid aerosols. No PFAS are therefore necessary as mist suppressants. ${ }^{50}$

Another reason why the use of chromium(VI) is not essential for all types of decorative chrome plating is that trivalent chromium can be used instead. ${ }^{47,51}$ One disadvantage is that trivalent chromium cannot achieve the silvery-bluish color of chromium(VI). ${ }^{51,52}$ However, trivalent chromium is much less toxic than chromium(VI) and mist-suppressing PFAS are therefore not needed in processes with trivalent chromium. ${ }^{42,47,53}$ A different technology for decorative chrome plating includes processes based on physical vapor deposition (PVD), where PFAS are also not needed. ${ }^{52}$ However, the quality of the coating produced by PVD may not be sufficient for materials used for drinking water contact. ${ }^{54}$ Also, as of 2016, PVD-based processes did not fulfill the requirements of the German automotive industry in terms of quality, aesthetic value and functionality of the parts. ${ }^{55}$ 
For hard metal plating, it has been suggested that trivalent chromium could also be used in some applications. ${ }^{47}$ Atotech launched a hard-chrome plating process with trivalent chromium and stated that the process exhibits the same benefits as hexavalent-chromium processes. ${ }^{56}$ This includes high plating speed, bath stability, and high hardness deposits and wear resistance. However, the technology requires a nickel underlayer to meet the corrosion resistance requirements and is therefore not a drop-in replacement. Besides Atotech, Faraday Technology is also working on a trivalent chromium plating process for functional applications. They stated to us that the trivalent chromium plating process has already passed the tests specified for aerospace applications. They are currently further optimizing the process such that the coating microstructure and appearance is equivalent to that of hexavalent-chromium coatings. More information on chrome plating with trivalent chromium is provided in the SI-1.

Alternatives Assessment. The use of closed systems with underpressure reduces the reproductive hazard of chromium(VI) from the level of "Repr. 1B/H360D" (may damage the unborn child) to "Repr. 2/H361f" (suspected of damaging fertility). It also reduces the environmental hazard as no PFAS or other hazardous substances are needed in this process.

The use of chromium(III) instead of chromium(VI) in electroplating constitutes a shift to a less hazardous substance without the carcinogenic, mutagenic and reproductive toxicity properties of chromium(VI). ${ }^{57}$ Atotech and Faraday Technology (the latter from 2015 on) use no boric acid in their processes, which was for a long time a serious drawback of the chromium(III) process. ${ }^{56,58}$

Processes based on PVD use UV-lacquer and, according to Gerhardi Kunststofftechnik (2016a), ${ }^{52}$ some of the substances in the application in UV-lacquers are listed as Substances of Very High Concern in the EU. However, no further details are available.

Conclusion. PFAS have been technically important in both decorative and hard chrome plating. For many decorative chrome plating processes, trivalent chromium can be used. For hard chrome plating, there are two ways in which the use of PFAS in the electroplating step may be avoided: either by switching to a closed system in a process still using hexavalent chromium or by switching to trivalent chromium, which removes the need for a mist-suppressing surfactant. The first approach has already been used for 10 years and is feasible, although initially more expensive due to the costs for modifications of the plant. The second approach is not yet used at large scale and might require more research and time for implementation. The use of PFAS in chrome plating (electroplating) is substitutable. Whether or not PFAS can be replaced in the etching pretreatment process is unclear.

3.5. Chemical-Driven Oil Production. Uses. The production of crude oil and gas generally occurs in three steps: (i) primary oil recovery (providing $12-15 \%$ of the oil), (ii) secondary oil recovery (or "water flooding", providing an additional $15-20 \%$ of the oil); (iii) enhanced oil recovery (EOR), aiming to collect some of the remaining $60-70 \%$ of the oil. ${ }^{59}$ EOR techniques are classified in thermal processes, gas injection, and chemical flooding. ${ }^{59}$

Fluorinated surfactants are used during water flooding (secondary oil recovery) and in EOR. ${ }^{60}$ When used in water and chemical flooding, fluorinated surfactants increase the permeability of the formation by reducing the interfacial tension between the reservoir surface and the aqueous phases. ${ }^{61}$ Fluorinated surfactants also increase the wettability of the rock. ${ }^{62}$ In chemical flooding, fluorinated surfactants are used to render the surfaces of the oil reservoirs hydrophobic and oleophobic. This supports the displacement of the oil from the sand and rock formations. ${ }^{63}$ Fluorinated surfactants are also used in fracturing rock formations penetrated by the wellbore, where they act as part of a foaming agent that initiates and extends the fractures in the formation. ${ }^{64}$ It has also been described that fluorinated surfactants are used to generate foam that is stable in contact with the crude oil, while imbibing and transporting the oil through the subterranean formation. $^{61}$

Overall, PFAS are used for several different functions in chemical-driven oil production. Generally, they are used because they have a very low surface tension (between 17 and $24 \mathrm{mN} / \mathrm{m}$ ) and are chemically and thermally stable. ${ }^{61}$

Availability of Alternatives. Multiple efforts have been made to synthesize alternative surfactants with the same physicochemical properties as PFAS. ${ }^{62}$ Branched hydrocarbon surfactants are an alternative which could replace fluorinated surfactants in EOR. Kiani et al. $(2019)^{62}$ studied a nonfluorinated anionic surfactant, iC18S(FO-180), CAS No. 181355-81-7, see structure in Figure 1A. C18S(FO-180) has a surface tension of around $25 \mathrm{nM} / \mathrm{m}$ and can enhance the oil recovery up to $72 \%{ }^{62}$

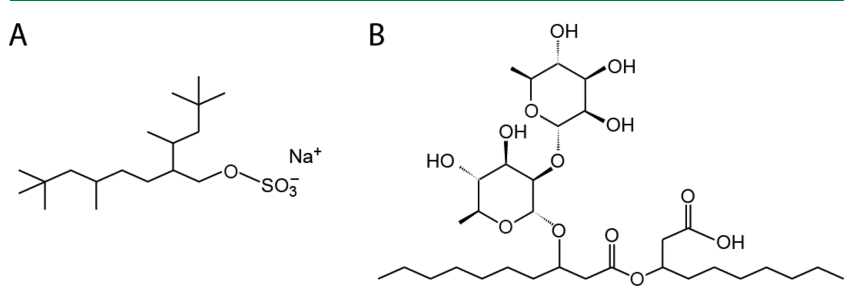

Figure 1. A: surfactant iC18S(FO-180), according to Alexander et al. (2014), ${ }^{65}$ Kiani et al. (2019); ${ }^{62}$ B: a rhamnolipid (CAS 4348-76-9) as an example of glycolipids used as biosurfactants.

Another potential additive in EOR is hydrophobin-II (HFBII). ${ }^{59}$ HFBII is a small cysteine-rich amphiphilic protein from the hydrophobin family (HFBs). HFBs are naturally produced by fungi and comprise about 100 amino acids. HFBs can self-assemble at hydrophilic-hydrophobic interfaces into an amphipathic film. This protein film renders hydrophobic surfaces of gas bubbles, liquids, or solid materials wettable, while hydrophilic surfaces can be turned hydrophobic. ${ }^{66}$ The ability of HFBs to self-assemble at oil-water interfaces and stabilize oil droplets makes them candidates for a PFAS-free EOR process. The company BASF has filed a patent for extracting hydrocarbons from oil sand with water and a hydrophobin. ${ }^{67}$ Another patent from BASF claims the use of hydrophobins as auxiliary-emulsifying agents for a drilling fluid. ${ }^{68}$ However, HFBII can only lower the air/water surface tension to $35 \mathrm{mN} / \mathrm{m}$, which is still too high for EOR. ${ }^{59}$ Blesic et al. $(2018)^{59}$ concluded that HFBII is not promising for EOR but also noted that an appropriate cosurfactant (e.g., mediumchain alcohols) may improve the performance of HFBII.

Beside hydrophobins there are other biosurfactants that have been used in microbial-enhanced oil recovery (MEOR). These include glycolipids (see Figure 1B), lipoproteins or lipopeptides, phospholipids, fatty acids or natural lipids, and particulate and polymeric biosurfactants. Examples for each group are given in the SI-1, Section 2.6. According to Varjani 
(2017), ${ }^{69}$ oil release from porous media may involve processes such as (a) dissolution of inorganic carbonates by bacterial metabolites, (b) production of bacterial gases that decrease the viscosity of oil, (c) generation of surface-active substances or wetting agents by some bacteria, and (d) high affinity of bacteria for solids, displacing oil by growing between oil and rock.

There is not much information about how well MEOR works in comparison to EOR with chemicals. However, MEOR has already been applied in the field. For example, in India the Oil and Natural Gas Corporation Limited have developed a technology for MEOC. ${ }^{70}$ The technology has been implemented in 130 oil wells and an encouraging success rate has been reported. ${ }^{70}$

In the U.S., $27 \%$ of the oil reservoirs and $40 \%$ of the oilproducing carbonate reservoirs may be suitable for MEOR. ${ }^{71}$ However, effective MEOR application may require substantial research on a case-by-case basis, because the environment will be unique with respect to soil and rock formation characteristics as well as physical and chemical conditions. ${ }^{71}$

Alternatives Assessment. Chemical identification was possible for surfactant iC18S. However, the substance does not have $\mathrm{C} \& \mathrm{~L}$ notifications or a REACH registration, which precluded an assessment of human-health hazards. Regarding environmental hazards of iC18S, EPI Suite estimates a low BCF (below 100) and a degradation half-life in water of 81 days. Whereas this half-life exceeds the EU's criteria for persistent substances (40 days in freshwater; 60 days in marine water), it is still much lower than the environmental degradation half-lives of PFAS.

Different amounts of data were found for different biosurfactants as identified by Varjani (2017). ${ }^{69}$ According to Banat $(1995)^{72}$ and Lazar et al. (2007), ${ }^{73}$ biosurfactants are biodegradable and have low toxicity, but data are scarce. There are studies available where no mutagenic effects and no reproductive toxicity were observed or expected for rhamnolipids, ${ }^{74}$ sophorolipids, ${ }^{75}$ and surfactin. ${ }^{76}$ Carcinogenicity data are lacking. Where biodegradation and toxicity data are available, they show that biosurfactants are readily biodegradable, ${ }^{74,75}$ or at least not persistent, ${ }^{77}$ with low aquatic toxicity. $^{74-76}$ There are limited data on bioaccumulation, but sophorolipids have a $\log K_{\mathrm{OW}}<4.5,^{75}$ suggesting that bioaccumulation is unlikely. For hydrophobin-II, no sufficient data for an assessment were found.

Conclusion. PFAS have been technically important in oil recovery. Alternatives are being explored and in some cases already in use. Therefore, PFAS can be replaced in the oil industry, but not equally well in all types of reservoirs and probably with research and development needed on a case-bycase basis. At least some of the PFAS uses are substitutable.

3.6. Processing Aids for Aqueous Emulsion Polymerization of Fluoropolymers. Uses. Fluoropolymers can be produced by several methods, including suspension polymerization, aqueous emulsion polymerization, solution polymerization, polymerization using supercritical $\mathrm{CO}_{2}$, and polymerization in the gas phase. The manufacturing process applied also depends on the commercial grade of the fluoropolymer that is manufactured (e.g., granular versus fine-powder PTFE). ${ }^{78}$ Patents for each of the main manufacturing processes are provided in the SI- 1 .

The most commonly employed polymerization methods include suspension polymerization and aqueous emulsion polymerization. Suspension polymerization generally does not involve (fluorinated) surfactants; it results in larger polymer particles than aqueous emulsion polymerization ${ }^{79}$ and is used, for example, for granular PTFE. ${ }^{78}$ Aqueous emulsion polymerization has traditionally involved the presence of a fluorinated surfactant, which is used to stabilize the polymer particles formed. ${ }^{79}$ Aqueous emulsion polymerization is used to produce fine-powder and dispersion products. $^{78,80}$

Availability of Alternatives. Fluoropolymer manufacturers are exploring novel processes to eliminate the use of PFAS in aqueous emulsion polymerization. For the production of polyvinylidene fluoride (PVDF), processes with fluorine-free emulsifiers have been implemented by multiple manufacturers. $^{81,82}$ One manufacturer has made this transition since $2008,{ }^{81}$ and its patents disclose varied processes that use fluorine-free alternative emulsifiers including blocks of polyethylene glycol, polypropylene glycol and/or polytetramethylene glycol, ${ }^{83-87}$ alkyl phosphonate, ${ }^{88}$ vinyl/acrylic acids, ${ }^{89}$ polyvinyl/acrylic acids, ${ }^{90}$ alkanesulfonates, ${ }^{91}$ siloxanes, ${ }^{92}$ and 3allyloxy-2-hydroxy-1-propanesulfonic acid salts. ${ }^{93}$ More information on the specific substances is provided in Section S2.7 in the SI-1. The same section in the SI- 1 also includes patents from several other manufacturers on fluorine-free emulsifiers in the polymerization of PVDF, but it is unclear whether or not they are actually in use.

Fluorine-free emulsifier-based processes for manufacturing other fluoropolymers, including fine-powder and dispersion PTFE, have been patented, ${ }^{94-98}$ but, to the best of our knowledge, are not yet implemented.

Alternatives Assessment. Some of the patents by Arkema from 2006 to $2020^{83-87}$ use multiple combinations of 14 glycol-based polymers as emulsifiers. The hazards of these emulsifiers would depend on which of the substances are used. As these are all polymers, EPI Suite could not be run and hazard data were collected from CLP and REACH registrations only. Of the seven substances with available data in CLP and REACH, four would constitute a reduction of hazards. However, there is also one substance that is on the $\mathrm{REACH}$ Authorization list because of endocrine-disrupting properties, one that is toxic to aquatic life with long-lasting effects and one that may damage fertility or the unborn child (for more information see SI-1 and SI-2).

All five patents from Arkema ${ }^{83-87}$ state that the invention "is generally practiced" with PEG, PPG, and/or PTMG as the sole emulsifiers. PPG and PEG have REACH registrations that provide evidence that the substances are not mutagenic, toxic to reproduction, toxic to the aquatic environment, persistent, or bioaccumulative. PPG also has evidence that it is not carcinogenic, whereas PEG has no evidence for this endpoint. PPG and PEG would be clear improvements in both humanhealth and environmental hazards. For PTMG there are insufficient data for an assessment. Overall, the use of a polyolefin glycol emulsifier most likely constitutes a reduction in human-health and environmental hazard. However, the patent mentions a variety of terminal groups that may be used in the polyolefin glycols. Because biodegradation of PEG and PPG needs alcoholic end groups, ${ }^{99}$ some of these end groups may impair the degradability of the substances.

The alternatives assessment of the 26 other emulsifiers patented by Arkema ${ }^{88-93}$ shows again that most of them would most likely constitute a reduction in human-health and environmental hazard. However, there are also substances among them that are very toxic to aquatic life with long-lasting 


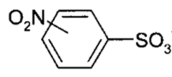

NBS

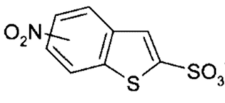

TBNO

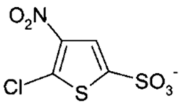

TN

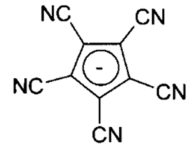

CN5

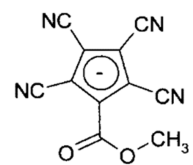

CN4-C1

Figure 2. Fluorine-free PAGs proposed by Glodde et al. (2010) ${ }^{101}$ and Liu et al. (2010). ${ }^{102}$

effects and one substance that is suspected of damaging fertility or the unborn child (for more information see SI-1 and SI-2). Again, it very much depends on which of the emulsifiers are actually used as patents tend to cover the broadest scope of possibilities.

Conclusion. PFAS emulsifiers have been technically important in aqueous emulsion polymerization. Alternatives have been developed for PVDF, but not yet for other fluoropolymers. The alternatives found for PVDF show that manufacturers need to carefully evaluate and manage their alternatives as some of the patented substances are toxic to aquatic life or toxic for reproduction. According to Arkema, during nearly 15 years of research, many alternatives have been studied; some only enabled a good polymerization process but were not able to meet the risk objectives and some were able to meet both, which proves that it is possible to produce PVDF with alternative emulsifiers exhibiting a good ecotoxicological profile. Beyond the question of PFAS emulsifiers, the essentiality of using fluoropolymers would also have to be assessed.

3.7. Semiconductor Industry. Overview. Semiconductor manufacturing is a multistep sequence of photolithography and chemical processing steps, comprising over 500 steps in total. ${ }^{47}$ Photolithography is the process by which the circuits are created on the semiconductor wafers. ${ }^{100}$ Here, a thin film of a photoresist (light-sensitive polymer) is first applied to a substrate, such as silicon-based wafers. Then, light is used to transfer a geometric pattern from a photomask to the photoresist on the wafer. The photoresist is altered when exposed to light, and this allows structures to be built up on the wafer. ${ }^{100}$ Photoresists require the presence of (fluorinated) photoacid generators (PAGs) that enable the etching of images smaller than the wavelength of visible light. ${ }^{47}$ Other uses of PFAS in the semiconductor industry include, e.g., developer and rinse solutions. More information is provided in the SI-1.

3.7.1. Photoacid Generators (PAGs). Uses. PAGs are components of a photoresist formulation that are able to generate strong acids under light irradiation. PAGs based on perfluorooctanesulfonic acid (PFOS) were used for a long time and then replaced by PAGs based on perfluorobutanesulfonic acid (PFBS) or PAGs based on functionalized fluoroethanesulfonates. ${ }^{101}$ However, these alternative substances are also PFAS, so there is still a need for fluorine-free alternatives.

Availability of Alternatives. One of several requirements in the design of a substance that can form a strong acid is the ability to delocalize the negative charge of the resulting anion. This can be achieved, for example, through the $\pi$-system of a benzene ring. The isomers of nitrobenzenesulfonate (NBS) are an example of such PAGs. ${ }^{101}$ Preliminary work has also shown that acceptor-substituted thiosulfonate anions exhibit good performances as strong acid generators. Specific examples include benzo[b]thiophene-2-sulfonic acid, 4(or 7)-nitro-, ion(1-) (TBNO) or 2-thiophenesulfonic acid, 5-chloro-4nitro-, ion(1-) (TN). Another possibility for strong acid generation are PAGs based on acceptor-substituted aromatic anions, such as pentacyanocyclopentiadienide (CN5) or methoxycarbonyl-tetracyanocyclopentadienide $(\mathrm{CN} 4-\mathrm{C} 1)^{102}$ (Figure 2).

Glodde et al. $(2010)^{101}$ and Liu et al. $(2010)^{102}$ evaluated some basic performance metrics of the five nonfluorinated PAG anions in photoresist formulations with triphenylsulfonium (TPS) and compared the results to photoresist formulations of perfluorobutanesulfonate as anion with TPS (TPS PFBS). The fluorine-free formulations showed promising results in optical clarity and thermal stability compared to TPS PFBS. However, the fluorine-free PAGs did not perform as well as the TPS PFBS with respect to sensitivity, line-width roughness, and resolution. Fluorine-free PAGs developed by IBM are described in various patents (see SI-1) and have also been officially announced. ${ }^{103}$

Alternatives Assessment. An assessment of alternatives could be carried out for the PAGs where the chemical structures are known. No REACH registrations were found for the corresponding CAS numbers, but C\&L notifications were found for two isomers of NBS. C\&L data for NBS indicated that this substance has no CMR properties. Environmental hazard data were estimated for NBS, TBNO, TN, and CN5, all of which indicate lower environmental hazard than for PFAS. Calculated degradation half-lives are between 21 and 42 days. All calculated BCF values are below 1 .

Conclusion. According to the estimated persistence and bioaccumulation potential of these compounds, the alternatives reviewed seem to be less hazardous than PFOS or PFBS and also seem to be capable of generating strong acids. However, as mentioned above, these fluorine-free PAGs have some technical limitations which are currently prohibitive to highvolume manufacturing. Thus, there is still a need for additional research and development of fluorine-free PAG alternatives. In addition to research focused on the replacement of PFAS with safe and effective nonfluorinated alternatives, research is also needed to develop new technologies for PFAS removal and destruction, as long as PFAS cannot be replaced in these applications. As the viability of photolithography chemicals requires the simultaneous satisfaction of multiple overlapping performance requirements, and the manufacture of semiconductors relies on many interlocking steps, the use of fluorine-free PAGs will require additional technological innovation and process adaptations to ensure viable and effective solutions that can be manufactured reliably.

3.7.2. Immersion Liquid, Developer Solution, and Rinse Solution. Uses. Besides use in photoresists, PFAS are also used in other parts of immersion lithography. A patent from the Taiwan Semiconductor Manufacturing Company ${ }^{104}$ describes the use of fluorinated surfactants as additives to developer and chemical rinse solutions. PFAS are used in these solutions to lower the contact angle of the solutions and, thus, reduce watermark defects after dry spinning. The employment of 
PFAS may also help to avoid pattern collapse during spindrying. ${ }^{104}$

Availability of Alternatives. A patent from $\mathrm{BASF}^{105}$ refers to the aforementioned patent US20080299487 $7^{104}$ and discloses that a new liquid (and a method of using it) for immersion photolithography of photoresist layers has been developed that allows for a high aspect ratio for line-space dimensions of $20 \mathrm{~nm}$ and below without causing pattern collapse, line edge roughness, and watermark defects, without the use of fluorinated surfactants. The patent remains vague regarding the alternative substance/s employed and describes only the possible anionic and cationic functional groups, see SI-1. However, it is mentioned that the newly developed fluorine-free liquid may be used as an immersion liquid for immersing photoresists during irradiation, as developer solution for photoresist layers, and as chemical rinse solution for rinsing the patterned material layers. ${ }^{105}$ It is also mentioned that the method does not only work for immersion photoresist layers, but also for extreme UV (EUV) and electron beam (eBeam) photoresist layers. EUV lithography recently became ready for mass production and is the most suitable candidate for the next pattern-feature size ( $5 \mathrm{~nm}$ node). ${ }^{106,107}$

Alternatives Assessment. No information is available on the hazards of the alternatives proposed.

Conclusion. It seems that the alternative described by Klipp et al. (2012) ${ }^{105}$ fulfills the technical functions needed and has potential also for future developments in the semiconductor industry. As such, it is a step toward the application of PFASfree alternatives, which may make PFAS in these uses substitutable. More information would be needed on the physicochemical properties and hazards of these materials.

Conclusion. Even though PFAS may currently be essential in some uses in the semiconductor industry, it has been recognized that PFAS are problematic for the environment and efforts are being made to replace PFAS with fluorine-free alternatives. ${ }^{108}$ The Semiconductor Industry Association reported that the semiconductor industry globally has successfully completed the phase-out of PFOS, ${ }^{47}$ but PFOS has often been replaced by other shorter-chain PFAS. The two examples above show that fluorine-free alternatives have been patented but that more research is needed to implement them.

\section{DISCUSSION}

The case studies illustrate the diversity and complexity of the task of a PFAS phase-out. In several cases, the technical function of PFAS directly derives from their water- and oilrepellency and it is relatively clear that PFAS can be replaced by PFAS-free water- or oil-repellent substances (bicycle lubricants, carpets, cleaning products). In chrome plating and fluoropolymer production, the technical function of PFAS is also well-defined, but the conditions and requirements are so demanding that finding alternatives is challenging. Finally, in the semiconductor industry and in oil recovery, PFAS have several different technical functions and/or are used in a multitude of process steps, which makes it more difficult to find suitable alternatives, and many different substances and/or process modifications may be needed.

4.1. Availability of Alternatives. An extensive search of the available literature was conducted. However, the list of identified alternatives is not exhaustive and there might be more (and maybe even better) alternatives. An important point regarding patents is that patents generally reflect an early stage of research and development and cover a broad range of options that might work in processes to be further developed. Therefore, they mostly do not describe solutions that are ready to be implemented, and further research and development over several years may be needed before a viable solution is available.

Alternatives that are already on the market were identified for four of the seven case studies: bicycle lubricants, carpets, household cleaning products, and chrome plating. We did not investigate industrial cleaning products as the specifications for these products might differ substantially between different use areas. The identified alternative products and processes in the four use areas will not be suitable for all specific uses in these areas. However, they show that innovation has happened and that there are feasible options to replace PFAS in these uses. The case of chrome-plating illustrates that sometimes a change in process conditions (underpressure) can be as effective as finding a chemical substitution.

Alternatives for processing aids in aqueous emulsion polymerization have been identified for PVDF but not for other fluoropolymers. However, there are numerous patents that describe fluorosurfactant-free polymerization processes for other fluoropolymers as well, suggesting that it is also possible to make fine-powder PTFE without fluorinated processing aids, but that it is more challenging than for PVDF. Developing PFAS-free emulsifiers requires extensive research and development and a long-term investment (probably more than 5 years). Furthermore, the solution found by one manufacturer will be patent-protected and not directly available for others, which also explains why, for example, in China perfluorooctanoic acid (PFOA) is still used.

The uses of PFAS in chemical-driven oil production and the semiconductor industry and the availability and suitability of alternatives are more difficult to assess. There are alternatives to PFAS also in chemical-driven oil production, but it is unclear how well they work compared to PFAS and what level of performance is necessary in each application. Substantial research might be needed on a case-by-case basis; ultimately, only technical experts in the area of oil drilling might be able to assess the alternatives. A similar situation occurs in the semiconductor industry. PFAS in the semiconductor industry are used in so many different steps and for so many different functions that it is difficult to judge the alternatives, in particular as processes build on each other and are very complex. However, the examples investigated here (PAGs and immersion liquid, developer solution, and rinse solution) show that there is awareness of the PFAS problem in the field and that research into alternatives is ongoing.

4.2. Alternatives Assessment. In the assessment of alternatives, we used a simple and pragmatic approach (information present in the C\&L and REACH databases, estimates from EPI Suite, where possible). A more detailed assessment would not have been feasible because of the large number of substances to be assessed and the lack of information about many of the substances. For an initial comparison for the purposes of illustrating the process and the data needs, the approach is sufficient.

The lack of publicly accessible information about many of the alternatives limits the scope of alternative assessments. For many substances, even the chemical identity was not known (and not revealed by the manufacturers contacted) and basic physicochemical properties and results from degradation and toxicity tests were lacking. In several cases, some qualitative information was available ("plant-based"; list of substance 
groups used such as polyolefin glycols or possible anionic and cationic groups), and for some substances, the chemical identity and structure were available so that some basic properties could be estimated.

Several alternatives were sufficiently characterized so that at least a partial comparison of their properties with those of the PFAS used could be performed. Often, the alternatives offer significant improvements compared to PFAS, at least with respect to persistence. However, it has to be reiterated that more chemical property and toxicity data for the alternatives need to be generated and made available in the public domain.

4.3. Transition to PFAS-Free Alternatives. For the consumer products investigated, the transition to PFAS-free alternatives is feasible without substantial problems. Alternatives are available and labeled and marketed as PFAS free or fluorine free. Regulatory requirements may help to transition to these alternatives. An example is the U.S. State of California, where they request carpet and rug manufacturers whose products contain PFAS to submit a Priority Product Notification (PPN) by August 30, 2021. ${ }^{109}$ Within 7 months after submitting the PPN, manufacturers need to remove the PFAS from the products.

In the area of chrome plating, a substantial step forward has been made and even in hard-chrome plating, where use of chromium(VI) was seen as a necessity, processes based on chromium(III) have been developed and are used by two manufacturers, ${ }^{56,110}$ and also for the process with chromium(VI), PFAS-free versions exist. However, approximately $22 \%$ of the authorizations under REACH are still for chrome plating with hexavalent chromium, ${ }^{111}$ which shows that more efforts are needed to support the transition.

In the areas of fluoropolymer production, oil recovery, and the semiconductor industry, the situation is more complex. Because of the demanding and highly diverse conditions or the many interlinked processes with many different PFAS uses, much broader assessments are required. In all three areas, the need for a replacement of PFAS has been recognized and research and development are underway. However, it is not clear to what extent and by how many manufacturers the alternatives are already in use and how much of the market is still based on processes using PFAS. In these areas, it may be desirable to establish technical expert committees that act as an interface between science/engineering and policy making. This would create greater transparency and make it easier for policy makers to follow the process and facilitate next steps. Stronger requirements to reduce emissions of PFAS-containing waste through, for example, stronger regulation and enforcement would increase the need to look for PFAS-free alternatives.

Finally, the transition requires better access to information about both PFAS uses and alternatives and their properties. For a group of chemicals of such high concern to environmental and human health, it is justified to require more transparency and publicly accessible data. What is needed at a minimum is information about the chemical identity and some minimum data on toxicity, degradability, and intended uses. This information should be available to the general public, and also within supply chains. A situation in which a product manufacturer receives a PFAS-free chemical from a chemical manufacturer and uses it in a consumer product without knowing the identity of the chemical, but just relies on the safety-data sheet, is not desirable.

Overall, the phase-out of PFAS may proceed on several different "tracks" with different time scales and priorities. One set of factors that determine these time scales and priorities are, obviously, the amounts of PFAS used and the extent to which the uses are open and dispersive. Another set of factors is the complexity of the assessment tasks and the amount of research and development needed. For a task of such high importance, but also complexity, a roadmap outlining these different tracks and corresponding timelines will be desirable.

\section{ASSOCIATED CONTENT}

\section{(I) Supporting Information}

The Supporting Information is available free of charge at https://pubs.acs.org/doi/10.1021/acs.est.1c03732.

A list of the manufacturers contacted, additional information on the case studies including information on an eighth case study (climbing ropes) (PDF)

MS Excel document listing data from the alternatives assessments (XLSX)

\section{AUTHOR INFORMATION}

\section{Corresponding Author}

Martin Scheringer - Institute of Biogeochemistry and Pollutant Dynamics, ETH Zürich, 8092 Zürich, Switzerland; RECETOX, Masaryk University, 62500 Brno, Czech Republic; (1) orcid.org/0000-0002-0809-7826; Email: scheringer@usys.ethz.ch

\section{Authors}

Juliane Glüge - Institute of Biogeochemistry and Pollutant Dynamics, ETH Zürich, 8092 Zürich, Switzerland; (1) orcid.org/0000-0003-1997-2750

Rachel London - Institute of Biogeochemistry and Pollutant Dynamics, ETH Zürich, 8092 Zürich, Switzerland

Ian T. Cousins - Department of Environmental Science, Stockholm University, SE-10691 Stockholm, Sweden; (1) orcid.org/0000-0002-7035-8660

Jamie DeWitt - Department of Pharmacology and Toxicology, Brody School of Medicine, East Carolina University, Greenville, North Carolina 27834, United States

Gretta Goldenman - Milieu Consulting SPRL, Brussels 1060, Belgium

Dorte Herzke - Norwegian Institute for Air Research (NILU), Fram Centre, N-9296 Tromsø, Norway, and Institute for Arctic and Marine Biology, UiT The Arctic University of Norway, N-9037 Tromsø, Norway

Rainer Lohmann - Graduate School of Oceanography, University of Rhode Island, Narragansett, Rhode Island 02882, United States; (1) orcid.org/0000-0001-8796-3229

Mark Miller - National Institute of Environmental Health Science and U.S. Public Health Service, Research Triangle Park, North Carolina 27709, United States

Carla A. Ng - Departments of Civil \& Environmental Engineering, Chemical \& Petroleum Engineering, and Environmental \& Occupational Health, University of Pittsburgh, Pittsburgh, Pennsylvania 15261, United States; (1) orcid.org/0000-0001-5521-7862

Sharyle Patton - Health and Environment Program, Commonweal, Bolinas, California 94924, United States

Xenia Trier - European Environment Agency, DK-1050 Copenhagen, Denmark

Zhanyun Wang - Chair of Ecological Systems Design, Institute of Environmental Engineering, ETH Zürich, 8093 Zürich, Switzerland; 이이.org/0000-0001-9914-7659 
Complete contact information is available at:

https://pubs.acs.org/10.1021/acs.est.1c03732

\section{Author Contributions \\ $\boldsymbol{\Delta}_{\text {J.G. and R.L. contributed equally. }}$}

\section{Notes}

The authors declare no competing financial interest.

\section{ACKNOWLEDGMENTS}

Helpful feedback on case study descriptions (bicycle lubricants, chrome plating, fluoropolymers, semiconductors) by representatives of Arkema, Faraday Technology, Fenwick's, IBM, and MacDermid is gratefully acknowledged. J.G. acknowledges funding from the Swiss Federal Office for the Environment. This work received funding from the European Union's Horizon 2020 research and innovation programme under the Marie Sklodowska-Curie grant agreement 860665 (PERFORCE3 Innovative Training Network). R. Lohmann acknowledges funding from the NIH Superfund Research Program (P42ES027706), and SERDP (ER18-1280 and ER201293). C.A.N. acknowledges funding from the National Science Foundation (grant 1845336). M.S. acknowledges funding by the CETOCOEN PLUS project (CZ.02.1.01/ $0.0 / 0.0 / 15003 / 0000469$ ), the project CETOCOEN EXCELLENCE (No CZ.02.1.01/0.0/0.0/17_043/0009632) and RECETOX RI (No LM2018121) financed by the Czech Ministry of Education, Youth and Sports. We thank A. Lindstrom (USEPA) for valuable contributions. This article has been supported by the Global PFAS Science Panel. We thank the Tides Foundation for support (grant 1907-59084).

\section{REFERENCES}

(1) Buck, R. C.; Franklin, J.; Berger, U.; Conder, J. M.; Cousins, I. T.; Voogt, P.; De; Jensen, A. A.; Kannan, K.; Mabury, S. A.; van Leeuwen, S. P. J. J. Perfluoroalkyl and Polyfluoroalkyl Substances in the Environment: Terminology, Classification, and Origins. Integr. Environ. Assess. Manage. 2011, 7 (4), 513-541.

(2) OECD. Toward a New Comprehensive Global Database of Perand Polyfluoroalkyl Substances (PFASs) - Series on Risk Management Nr.. 39.

(3) Fenton, S. E.; Ducatman, A.; Boobis, A.; DeWitt, J. C.; Lau, C.; $\mathrm{Ng}$, C.; Smith, J. S.; Roberts, S. M. Per- and Polyfluoroalkyl Substance Toxicity and Human Health Review: Current State of Knowledge and Strategies for Informing Future Research. Environ. Toxicol. Chem. 2021, 40 (3), 606-630.

(4) Cousins, I. T.; Goldenman, G.; Herzke, D.; Lohmann, R.; Miller, M.; Ng, C. A.; Patton, S.; Scheringer, M.; Trier, X.; Vierke, L.; Wang, Z.; DeWitt, J. C. The Concept of Essential Use for Determining When Uses of PFASs Can Be Phased Out. Environ. Sci. Process. Impacts 2019, 21, 1803-1815.

(5) Cousins, I. T.; Ng, C.; Wang, Z.; Scheringer, M. Why Is High Persistence Alone a Major Cause of Concern? Environ. Sci. Process. Impacts 2019, 21, 781-792.

(6) Cousins, I. T.; DeWitt, J. C.; Glüge, J.; Goldenman, G.; Herzke, D.; Lohmann, R.; Ng, C. A.; Scheringer, M.; Wang, Z. The High Persistence of PFAS Is Sufficient for Their Management as a Chemical Class. Environ. Sci. Process. Impacts 2020, 22, 2307-2312.

(7) Sweden_and_other_EU_countries. Elements for an EU-Strategy for PFASs, 2019.

(8) EC. Commission Staff Working Document - Poly- and Perfluoroalkyl Substances (PFAS) Accompanying the Document "Communication from the Commission to the European Parliament, the Council, the European Economic and Social Committee and the Committee of the Region, 2020.
(9) Fernández, S. R.; Kwiatkowski, C.; Bruton, T. Building A Better World - Eliminating Unnecessary PFAS in Building Materials, 2021.

(10) Holmquist, H.; Schellenberger, S.; van der Veen, I.; Peters, G. M.; Leonards, P. E. G.; Cousins, I. T. Properties, Performance and Associated Hazards of State-of-the-Art Durable Water Repellent (DWR) Chemistry for Textile Finishing. Environ. Int. 2016, 91, 251264.

(11) DEPA. Alternatives to Perfluoroalkyl and Polyfluoroalkyl Substances (PFAS) in Textiles, 2015.

(12) Trier, X.; Taxvig, C.; Rosenmai, A. K.; Pedersen, G. A.PFAS in Paper and Board for Food Contact - Options for Risk Management of Poly- and Perfluorinated Substances; Copenhagen, Denmark, 2017.

(13) IPEN. Fluorine-Free Firefighting Foams (3F) Viable Alternatives to Fluorinated Aqueous Film-Forming Foams (AFFF); 2018.

(14) KEMI Swedish Chemicals Agency. Occurrence and Use of Highly Fluorinated Substances and Alternatives; 2015.

(15) Poulsen, P. B.; Jensen, A. A.; Wallström, E. More Environmentally Friendly Alternatives to PFOS Compounds and PFOA, 2005.

(16) Glüge, J.; Scheringer, M.; Cousins, I. T.; DeWitt, J. C.; Goldenman, G.; Herzke, D.; Lohmann, R.; Ng, C. A.; Trier, X.; Wang, Z. An Overview of the Uses of Per- and Polyfluoroalkyl Substances (PFAS). Environ. Sci. Process. Impacts 2020, 22, 1462-1468.

(17) Pan, Y.; Zhang, H.; Cui, Q.; Sheng, N.; Yeung, L. W. Y.; Guo, Y.; Sun, Y.; Dai, J. First Report on the Occurrence and Bioaccumulation of Hexafluoropropylene Oxide Trimer Acid: An Emerging Concern. Environ. Sci. Technol. 2017, 51 (17), 9553-9560. (18) Gebbink, W. A.; Van Asseldonk, L.; Van Leeuwen, S. P. J. Presence of Emerging Per- and Polyfluoroalkyl Substances (PFASs) in River and Drinking Water near a Fluorochemical Production Plant in the Netherlands. Environ. Sci. Technol. 2017, 51 (19), 11057-11065.

(19) Lindstrom, A. B.; Galloway, J. E.; Strynar, M. J.; Knappe, D.; Sun, M.; Newton, S.; Weavers, L. K. Emerging Per- and Polyfluoroalkyl Substances (PFAS). Highly Fluorinated Compounds Social and Scientific Discovery; Northeastern University Social Science Environmental Health Research Institute, Boston. 2017.

(20) Galloway, J. E.; Moreno, A. V. P.; Lindstrom, A. B.; Strynar, M. J.; Newton, S.; May, A. A.; May, A. A.; Weavers, L. K.; Weavers, L. K. Evidence of Air Dispersion: HFPO-DA and PFOA in Ohio and West Virginia Surface Water and Soil near a Fluoropolymer Production Facility. Environ. Sci. Technol. 2020, 54 (12), 7175-7184.

(21) Lin, A. Y. C.; Panchangam, S. C.; Lo, C. C. The Impact of Semiconductor, Electronics and Optoelectronic Industries on Downstream Perfluorinated Chemical Contamination in Taiwanese Rivers. Environ. Pollut. 2009, 157 (4), 1365-1372.

(22) Tang, C. Y.; Fu, Q. S.; Robertson, A. P.; Criddle, C. S.; Leckie, J. O. Use of Reverse Osmosis Membranes to Remove Perfluorooctane Sulfonate (PFOS) from Semiconductor Wastewater. Environ. Sci. Technol. 2006, 40 (23), 7343-7349.

(23) US EPA. Estimation Programs Interface Suite for Microsoft®Windows. US Environmental Protection Agency: Washington DC, 2012.

(24) BikeRadar. Chain lube buyer's guide: what's the best chain lube for your bike? https://www.bikeradar.com/advice/buyers-guides/ chain-lubes/ (accessed 2020/10/28).

(25) Maples, P. D. Dry Lubricant. Patent US5898022, 1999.

(26) Dy-Mark. Safety Data Sheet for Dy-Mark Protech Wet PTFE Lubricant, 2016.

(27) Smith, J. Personal Communication with Jon Smith from Fenwicks Bike, 2021.

(28) Transa. Green Oil https://www.transa.ch/marken/green-oil/.

(29) GreenOil. Britain's first bike biolube https://www.green-oil. net/products.html.

(30) WPL. Lubricate https://us.wplbike.com/collections/lubricate (accessed 2020/10/28).

(31) WPL. Bio-Based vs Biodegradable Bicycle Maintenance Products https://us.wplbike.com/blogs/news/bio-based-vsbiodegradable-bicycle-maintenance-products.

(32) HBN. Eliminating Toxics in Carpet: Lessons for the Future of Recycling, 2017. 
(33) Interface. Stain Resistance https://www.interface.com/US/enUS/sustainability/product-transparency/Stain-Resistance-PFAS-en_ US.

(34) Econyl. Econyl Stay Clean http://www.econyl.com/stay-clean/

(35) Nordic Council. Hazardous Substances in Plastics - Ways to Increase Recycling, 2017.

(36) Onyshko, J.; Hewlett, R.Toxics in Carpets in the European Union, 2018.

(37) Blue-Angels. Basic Award Criteria for Floor Coverings https:// www.blauer-engel.de/en/products/construction-heating/floorcoverings-textile/floor-coverings.

(38) Cradle-to-cradle. Introducing the Cradle to Cradle Certified Product Standard Version 4.0 https://www.c2ccertified.org/getcertified/cradle-to-cradle-certified-version- 4 .

(39) GUT. Prüfkriterien 2020 http://docplayer.org/214201900Gut-pruefkriterien-2020.html.

(40) OEKO-TEX. Annex 4: Limit Values Table; 2021.

(41) Blauer Engel. Condor Group PA6-111-FS https://www. blauer-engel.de/en/products/construction-heating/bodenbelaegetextil/condor-group-pa6-111-fs (accessed 2019/6/12).

(42) POPRC. Consolidated Guidance on Alternatives to Perfluorooctane Sulfonic Acid and Its Related Chemicals (UNEP/POPS/ POPRC.12/INF/15/Rev.1), 2016.

(43) Blom, C.; Hanssen, L. Analysis of Per- and Polyfluorinated Substances in Articles (M-360), 2015.

(44) Ecover. ZERO Dish Soap https://us.ecover.com/products/ zero-dish-soap/.

(45) Ecover. Laundry Detergent https://us.ecover.com/products/ laundry-detergent-lavender-field/.

(46) Ecover. Rinse Aid https://us.ecover.com/products/rinse-aid/. (47) POPRC. Report on the Assessment of Alternatives to Perfluorooctane Sulfonic Acid, Its Salts and Perfluorooctane Sulfonyl Fluoride (UNEP/POPS/POPRC.14/INF/13), 2019.

(48) Wiethoelter, D. Personal Communication with Dirk Wiethoelter from MacDermid, 2021.

(49) Poulsen, P. B.; Gram, L. K.; Jensen, A. A.; Rasmussen, A. A.; Ravn, C.; Müller, P.; Jorgensen, C. R.; Lokkegaard, K. Substitution of PFOS for Use in Nondecorative Hard Chrome Plating, 2011.

(50) Blepp, M.; Willand, W.; Weber, R. Verwendung von PFOS in der Galvanik - Kennzeichen eines geschlossenen Kreislaufs, Verwendung von Ersatzstoffen (63/2016), 2016.

(51) Hauser, H.; Füglister, L.; Scheffelmaier, T. Verwendung von Fluortensiden in der Galvanikbranche, 2020.

(52) Gerhardi Kunststofftechnik.Analysis of Alternatives for Chromium Trioxide - Non-Confidential Report, 2016.

(53) USEPA. Chromium Compounds - Factsheet, 2000.

(54) Grohe. Comments and Response to Comments on Authorisation of Chromium Trioxide, 2016.

(55) Gerhardi Kunststofftechnik. Comments and Response to Comments on Authorisation of Chromium Trioxide, 2016.

(56) Atotech. Atotech launches the first trivalent chromium hard chrome https://www.atotech.com/atotech-launches-the-firsttrivalent-chromium-hard-chrome-general-metal-finishing/ (accessed 2019/5/15)

(57) ECHA. Opinion on an Application for Authorisation for Chromium Trioxide Use: Functional Chrome Plating; Committee for Risk Assessment (RAC) and Committee for Socio-economic Analysis (SEAC), 2016.

(58) Hall, T. D.; Snyder, S. T.; Taylor, E. J.; Inman, M. E.; Xu, J.; Radhakrishnan, R. Development of a Functional REACH Compliant Trivalent Chromium Electroplating Process, 2017.

(59) Blesic, M.; Dichiarante, V.; Milani, R.; Linder, M.; Metrangolo, P. Evaluating the Potential of Natural Surfactants in the Petroleum Industry: The Case of Hydrophobins. Pure Appl. Chem. 2018, 90 (2), 305-314.

(60) Kissa, E. Fluorinated Surfactants and Repellents; Marcel Dekker AG, 2001.
(61) Murphy, P. M.; Hewat, T. Fluorosurfactants in Enhanced Oil Recovery. Open Pet. Eng. J. 2008, 1 (1), 58-61.

(62) Kiani, S.; Rogers, S. E.; Sagisaka, M.; Alexander, S.; Barron, A. R. A New Class of Low Surface Energy Anionic Surfactant for Enhanced Oil Recovery. Energy Fuels 2019, 33, 3162-3175.

(63) Bond, D. C. Increasing the Recovery of Petroleum from Wells. US2765851, 1956.

(64) Scherubel, G. A. Subterranean-Formation Acidizing with Foam. GB2018863, 1979.

(65) Alexander, S.; Smith, G. N.; James, C.; Rogers, S. E.; Guittard, F.; Sagisaka, M.; Eastoe, J. Low-Surface Energy Surfactants with Branched Hydrocarbon Architectures. Langmuir 2014, 30 (12), 3413-3421.

(66) Wösten, H. A. B.; Scholtmeijer, K. Applications of Hydrophobins: Current State and Perspectives. Appl. Microbiol. Biotechnol. 2015, 99 (4), 1587-1597.

(67) Bode, A.; Huff, J.; Guzmann, M.; Wuensch, J. R.; Subkowski, T.; Stein, S.; Karos, M.; Scholtissek, M.; Bechtloff, B.; Baus, U.; Bollschweiler, C. Extraction and Recovery of Petroleum from Oil Sand Using Hydrophobins and Derivatives. CA2642375, 2009.

(68) Guzmann, M.; Liu, Y.; Baus, U. Hydrophobins and Hydrophobin Fusion Proteins as Emulsifiers and Additives in Drilling Fluids. WO2006103253, 2006.

(69) Varjani, S. J. Biosurfactants in Microbial Enhanced Oil Recovery (Chapter 23). InModern Tools and Techniques to Understand Microbes; Varma, A., Sharma, A. K., Eds.; Springer, 2017; pp 369-380 DOI: 10.1007/978-3-319-49197-4.

(70) OTBL. MEOR (Microbial Enhanced Oil Recovery) Technology http://www.otbl.co.in/MEOR.php (accessed 2020/4/23).

(71) Singh, A.; Van Hamme, J. D.; Ward, O. P. Surfactants in Microbiology and Biotechnology: Part 2. Application Aspects. Biotechnol. Adv. 2007, 25 (1), 99-121.

(72) Banat, I. M. Biosurfactants Production and Possible Uses in Microbial Enhanced Oil Recovery and Oil Pollution Remediation: A Review. Bioresour. Technol. 1995, 51 (1), 1-12.

(73) Lazar, I.; Petrisor, I. G.; Yen, T. F. Microbial Enhanced Oil Recovery (MEOR). Pet. Sci. Technol. 2007, 25 (11), 1353-1366.

(74) US EPA. Biopesticides Registration Action Document Rhamnolipid Biosurfactant (PC Code 110029); 2004.

(75) ECHA. Sophorolipids: fermentation products of glucose and fatty acids, C18 (unsaturated), glycerol esters with yeast Candida Bombicola, partially hydrolysed https://echa.europa.eu/de/ registration-dossier/-/registered-dossier/12734/1/1.

(76) Hwang, Y. H.; Park, B. K.; Lim, J. H.; Kim, M. S.; Song, I. B.; Park, S. C.; Yun, H. I. Evaluation of Genetic and Developmental Toxicity of Surfactin C from Bacillus Subtilis BC1212. J. Health Sci. 2008, 54 (1), 101-106.

(77) Rodríguez-López, L.; Rincón-Fontán, M.; Vecino, X.; Moldes, A. B.; Cruz, J. M. Biodegradability Study of the Biosurfactant Contained in a Crude Extract from Corn Steep Water. J. Surfactants Deterg. 2020, 23 (1), 79-90.

(78) Introduction to Fluoropolymers (Chapter 6). In Introduction to Fluoropolymers; Ebnesajjad, S., Ed.; Elsevier Inc., 2013 DOI: 10.1016/ B978-1-4557-7442-5.00006-1.

(79) Hintzer, K.; Jurgens, M.; Kaspar, H.; Lochhaas, H.; Maurer, A. R.; Zipplies, T. Aqueous Emulsion Polymerization of Fluorinated Monomers Using a Perfluoropolyether Surfactant. US20070015865, 2007.

(80) Manufacturing Polytetrafluoroethylene (Chapter 7). In Introduction to Fluoropolymers; Ebnesajjad, S., Ed.; Elsevier Inc., 2013.

(81) Kynar500FSF. Fluorosurfactant Free Kynar® PVDF Resin https://www.kynar500.com/en/product-information/ fluorosurfactant-free/.

(82) Solvay. Solvay Launches Non-Fluorosurfactant Technologies in the U.S. https://www.solvay.com/en/press-release/solvay-launchesnon-fluorosurfactant-technologies-in-us.

(83) Amin-Sanayei, R.; Olmstead, C. Aqueous Process for Making Fluoropolymers. US20060281845, 2006. 
(84) Amin-Sanayei, R.; Durali, M.; Kappler, P.; Burch, G. Aqueous Process for Making Fluoropolymers. US20070135546, 2007.

(85) Amin-Sanayei, R.; Olmstead, C. Aqueous Process for Making a Stable Fluoropolymer Dispersion. US20070082993, 2007.

(86) Amin-Sanayei, R.; Durali, M.; Kappler, P.; Burch, G. Aqueous Process for Making Polyvinylidene Fluoride Dispersion. US20120142858, 2012.

(87) Goldbach, J. T.; Bonnet, A.; Kahn, A. P. Making a Fluoropolymer Dispersion. WO2020101963, 2020.

(88) Durali, M.; Hedhli, L.; Wille, R. Polymerization of Fluoropolymers Using Alkyl Phosphonate Surfactants. US20070032591, 2007.

(89) Durali, M.; Hedhli, L. Method of Producing Fluoropolymers Using Acid-Functionalized Monomers. WO2012030784, 2012.

(90) Durali, M.; Hedhli, L.; Amin-Sanayei, R. Polymerization of Fluoropolymers Using Non-Fluorinated Surfactants. WO2007018783, 2007.

(91) Wille, R. A.; Durali, M.; Hedhli, L.; Amin-Sanayei, Ramin Schmidhauser, J. Thermoplastic Fluoropolymers and Their Production Using Alkyl Sulfonate Surfactants. US20050239983, 2005.

(92) Wille, R. A.; Durali, M.; Hedhli, L.; Antoun, S. Y. Polymerization of Halogen-Containing Monomers Using Siloxane Surfactant and Aqueous Composition. EP1462461, 2004.

(93) Wille, R. A.; Hedhli, L.; Durali, M.; Antoun, S. Y. Polymerization of Fluoromonomers Using a 3-Allyloxy-2-Hydroxy1-Propane Sulfonic Acid Salt as Surfactant. EP1475395, 2004.

(94) Lyons, D. F. Production of Fluoroelastomers by Radical Emulsion Polymerization in the Presence of Anionic Phosphate Ester Surfactants. US20080262177, 2008.

(95) Tang, P. L. A Semi-Batch Emulsion Polymerization Process for Making Fluoroelastomers Using a Hydrocarbon Anionic Surfactant Introduced Shortly after Polymerization Has Started. US20080125558, 2008.

(96) Bissinger, P.; Dadalas, M. C.; Hintzer, K.; Mayer, L. S., Werner Zipplies, T. C. Preparation and Stabilization of Fluoropolymer Dispersions by Aqueous Emulsion Polymerization with Carbosilane Surfactants. US7678859, 2010.

(97) Zipplies, T. C.; Hintzer, K.; Dadalas, M. C.; Frey, O.; Lochhaas, K. H. Fluoropolymer Compositions Containing a Polyol Compound as Emulsifier and Methods of Making Them. WO2011014715, 2011.

(98) Brothers, P. D.; Gangal, S. V.; Khasnis, D. D. Employing Polyalkylene Oxides for Nucleation in Aqueous Polymerization of Fluoromonomer. US20150148481, 2015.

(99) Kawai, F. Biodegradation of Polyethers (Polyethylene Glycol, Polypropylene Glycol, Polytetramethylene Glycol, and Others). In Biopolymers Online; Matsumura, S., Steinbüchel, A., Eds.; Wiley, 2005 DOI: $10.1002 / 3527600035 . b p o l 9012$.

(100) Brooke, D.; Footitt, A.; Nwaogu, T. A.Environmental Risk Evaluation Report: Perfluorooctanesulphonate (PFOS), 2004.

(101) Glodde, M.; Liu, S.; Varanasi, P. R. Fluorine-Free Photoacid Generators for 193-nm Lithography Based on Non-Sulfonate Organic Superacids. J. Photopolym. Sci. Technol. 2010, 23, 173-184.

(102) Liu, S.; Glodde, M.; Varanasi, P. R. Design, Synthesis, and Characterization of Fluorine-Free PAGs for 193-nm Lithography. Proc. SPIE 2010, 7639 (March2010), 76390D.

(103) IBM. A Commitment to Environmental Leadership, 2010.

(104) Chang, C.-Y. Lithography Material and Lithography Process. US20080299487, 2008.

(105) Klipp, A.; Oetter, G.; Montero Pancera, Sabrina Honciuc, A.; Bittner, C. Method for Manufacturing Integrated Circuit Devices, Optical Devices, Micromachines and Mechanical Precision Devices Having Patterned Material Layers with Line-Space Dimensions of 50 $\mathrm{nm}$ and Less. WO2012127342, 2012.

(106) WikiChip. $7 \mathrm{~nm}$ lithography process https://en.wikichip.org/ wiki/7 nm lithography process (accessed 2020/7/17).

(107) Hatakeyama, J.; Ohashi, M. Resist Composition and Patterning Process. US20200073237, 2020.
(108) Glodde, M.; Liu, S.; Popova, I. Ionic, Organic Photoacid Generators for DUV, MUV and Optical Lithography Based on Peraceptor-Substituted Aromatic Anions. WO2009087027, 2009.

(109) DTSC. Carpets and rugs with perfluoroalkyl or polyfluoroalkyl substances (PFASs) as priority products https://dtsc.ca.gov/scp/ carpets-and-rugs-with-perfluoroalkyl-and-polyfluoroalkyl-substancespfass/.

(110) USEPA. Faraday Inc. Develops New Durable Trivalent Plating Process https://19january2017snapshot.epa.gov/sbir/faraday-incdevelops-new-durable-trivalent-plating-process .html (accessed 2020/10/28)

(111) ECHA. Adopted opinions and previous consultations on applications for authorisation https://echa.europa.eu/applicationsfor-authorisation-previous-consultations. 